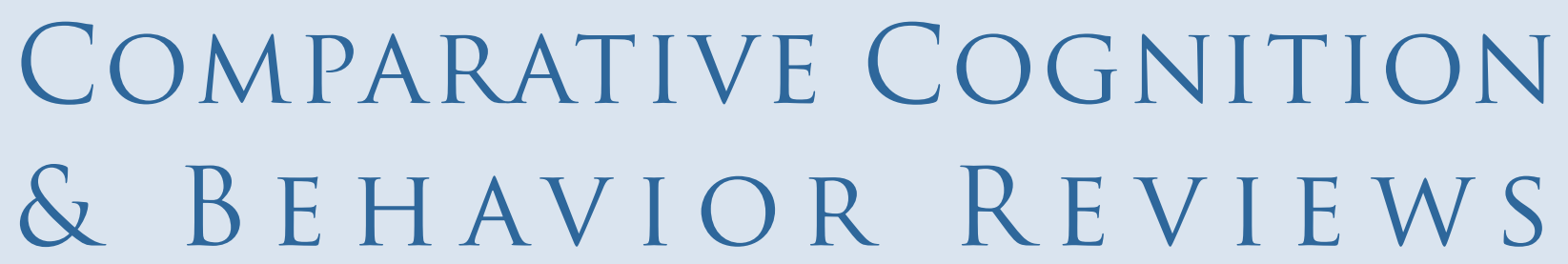

Factors That May Affect the Success of Scent Detection Dogs: Exploring Nonconventional Models of Preparation and Deployment

\author{
Sarah-Elizabeth Byosiere \\ School of Psychology and Public Health, La Trobe University, \\ Bendigo, Australia \\ Department of Psychology, Hunter College, \\ City University of New York
}

\author{
Lynna C. Feng and Nicholas J. Rutter \\ School of Psychology and Public Health, La Trobe University, \\ Bendigo, Australia
}

In the target article titled "Behavioral and Cognitive Factors That Affect the Success of Scent Detection Dogs," Troisi and colleagues highlight several challenges associated with scent detection dog preparation and deployment. Traditionally, scent detection dogs have been bred, raised, and trained at designated training facilities. More recently, several organizations, primarily in the conservation detection industry, have employed nonconventional models of scent detection dog selection and preparation. In this commentary, we highlight three of these nonconventional models: the community-based model, in which community members and their privately owned dogs are trained for deployment; the community-fostered model, in which puppies live with foster families during training; and the shelter-based model, in which dogs are sourced from shelters and rescues. We generally discuss these approaches and emphasize various benefits for both the dogs and the organizations. Finally, we explore the possibility that different models of scent detection selection, preparation, and deployment may support scientific opportunities for a better understanding of the ideal qualities of a detection dog-handler team.

Keywords: scent detection dogs, preparation, deployment, nonconventional approach, human-dog interactions

In the target article "Behavioral and Cognitive Factors That Affect the Success of Scent Detection Dogs," Troisi, Mills, Wilkinson, and Zulch (2019) provide a review on the current state of research in scent detection dogs. Specifically, the authors highlight important factors underlying preparation and deployment of scent detection dogs that may influence individual variation within the population, as well as task-specific learning. As noted in the target article, dogs can be trained to detect a variety of scents (biological and nonbiological), which has led to the use and implementation of a variety of different preparation and deployment procedures (for an in-depth review, see Browne, Stafford, \& Fordham, 2006). Even though these procedures are often 
specialized for the type of scent detection task a dog is trained for, there remain specific concerns regarding the preparation and deployment of scent detection dogs in general. Previous studies have reported that many working dogs fail to complete training successfully and are released either during training (Evans, Herbold, Bradshaw, \& Moore, 2007) or at the end of training (Maejima et al., 2007; Wilsson \& Sundgren, 1997). This commonly observed "wastage effect" not only economically affects organizations but also can result in welfare concerns on behalf of the dogs (Cobb, Branson, McGreevy, Lill, \& Bennett, 2015).

Most likely due to the currently available empirical evidence, the target article primarily focuses on relevant research for scent detection preparation and deployment procedures in which dogs are bred and/or selectively purchased and are reared, trained, and housed at their respective organizations until deployment. However, nonconventional models of preparation and deployment do exist. In an attempt to embrace the authors' recommendation of encouraging cooperation and synergy between academics and professionals (Troisi et al., 2019), we discuss a variety of nonconventional approaches to the preparation and deployment of scent detection dogs. Using the target article as a framework, we aim to briefly highlight some of these nonconventional models, stressing the potential benefits they may have for the dogs, the handlers and the organizations, and the scientific community.

\section{Community-Based Model}

A number of detection dog models exist in which individuals train their privately owned dogs for detection work. For the purposes of this commentary, we call this the community-based model. Generally, these dogs live with their owner, and therefore programs that use this approach are typically not responsible for rehoming dogs that may be unsuccessful in their training.

Authors' Note: Sarah-Elizabeth Byosiere, Department of Psychology, Hunter College, 695 Park Ave, New York, NY 10065.

Correspondence concerning this article should be addressed to Sarah-Elizabeth Byosiere at sarah-elizabeth.byosiere@hunter .cuny.edu

Acknowledgments: All authors contributed equally.
Various differences exist within community-based approaches. Some have mentor trainers who guide and support owners so that owners conduct all of the handson training and handle their dogs during deployment. Other programs allow only experienced trainers to prepare the dogs, but owners are involved in handling procedures. Still others have dedicated trainers who work exclusively with the dogs so that owners do not participate in training or deployment. Moreover, the specifics of community-based approaches can differ. For some programs, the trained dogs may have been initially sourced as companion animals. These dogs have varying socialization and training backgrounds and temperaments, and they are secondarily involved in detection work. In other cases, owners may purposefully select and acquire a dog based on desirable characteristics specifically for scent work.

Perhaps the most widely recognized communitybased approach is that used by search-and-rescue organizations. This well-established approach typically deploys volunteer dog-handler teams after they have completed relevant training and certification. Although these organizations do not have a training regimen, resources are available to help with advice regarding dog selection, training, equipment, mentoring, and annual workshops. Comparably, the Conservation Dogs Programme at the New Zealand Department of Conservation (n.d.) allows prospective dog-handler teams, with experience and relevant qualifications, to apply for governmental certification. Once certified, volunteer dog-handler teams can be deployed in the field.

In addition to this certification-style approach to the community-based model, another involves recruiting and training volunteers and their companion dogs for scent detection work. This approach has generally been applied to conservation detection dogs (for a review of scent detection in conservation settings, see Beebe, Howell, \& Bennett, 2016). Once successful, dog-handler teams generally conduct surveys in the field. However, is important to note that some programs use laboratorybased detection tasks, field-based detection tasks, or a combination of the two. Some programs that use such a community-based model include, but are not limited to, the Cape Otway Conservation Ecology Centre's Otways Conservation Dogs program, the La Trobe Conservation Detection Dog program, Detection Dogs for Conservation at the University of the Sunshine Coast, Waikato University's scent-detection program, and the Canid and Reptile Behaviour and Olfaction Team. 


\section{Benefits to the Dogs and Organizations}

There are several benefits for dogs and organizations using the community-based model. As emphasized in the target article's section named "Housing and General Management Effects on Learning and Performance," certain living conditions may affect learning (Rooney, Clark, \& Casey, 2016; Troisi et al., 2019). Typically, dogs in community-based models, especially those sourced primarily for a companionship role, live with their owner in a home-based environment. Home environments are usually characterized by comparatively less noise than kennels, which the target article highlighted as potentially detrimental to a dog's hearing and therefore readiness to respond to cues. Home environments may also provide increased opportunities for socialization with owners compared to kennels, which may help foster a closer working relationship between the dog and handler and influence search dog performance (Jamieson, Baxter, \& Murray, 2018). Particularly for cases in which dogs are deployed irregularly (e.g., following a natural disaster), it is possible for dogs to live in a home environment while they are not working rather than living in a kennel. Thus, the welfare requirements of dogs in community-based models may be more easily ensured than in traditional models.

There are several benefits of a community-based approach for organizations that select, train, or deploy scent detection dogs. Unlike traditional models, community-based models do not incur extensive costs related to breeding or sourcing dogs to recruit into a training program. Furthermore, once recruited, dogs continue to live with their owners, meaning that their owners can continue to cover costs associated with housing, food, and veterinary care. Training-related costs can also be minimized compared to traditional approaches, especially if paid professionals provide training advice but owners conduct the training and practice on their own time. Finally, having volunteer owners deploy their dogs can also reduce the cost of deployment. As such, taking advantage of a community-based model could make it feasible for organizations to develop large-scale doghandler teams with limited resources.

Although the relative success rate of dogs from a community-based model is unknown compared with a more traditional model, any unsuccessful dogs can return to their role of companionship without needing to be rehomed by the organization. Therefore, a communitybased model, focused on minimizing resources dedicated to the preparation of each individual scent detection dog, could ultimately reduce the wastage of time and financial resources invested into unsuccessful dogs.

\section{Community-Fostered Models}

Another nonconventional approach, which we term the community-fostered model, involves institutional structure supplemented by community involvement. Under this model, a professional training organization places puppies into local community foster homes. Community members are generally responsible for socializing and teaching the puppy basic manners, and professional trainers conduct specialized task training.

One organization currently implementing the community-fostered model is the Penn Vet Working Dog Group (Penn Vet, n.d.). Beginning at 8 weeks of age, puppies are enrolled in the Working Dog Center Puppy Foundation Program. Each puppy lives with a foster family who drops the puppy off at the Working Dog Center on weekdays for training in specialized skills such as live human search, agility, and obedience, in addition to scent detection training.

\section{Benefits to the Dogs and Organizations}

Similar to the community-based model, dogs in community-fostered models benefit from various housing and general management effects. As dogs in training are picked up and dropped off for training, they do not live full-time in an on-site kennel environment. This means that puppies' development can occur in a home environment, with a foster family to facilitate positive experiences with everyday sounds and situations that the dogs will likely encounter in their future working roles. This model can also maximize the amount of time that puppies spend with human caretakers, beyond what would be economically or logistically feasible for staff in a kennel facility. Thus, dogs and handlers may benefit both directly and indirectly from various socialization practices.

Training organizations implementing a communityfostered model can also minimize the resources required for animal housing facilities and care outside of regular working hours. It is likely that by limiting these resources, organizations implementing this approach are more economically viable. Although many organizations implementing this approach still cover the cost of food and vet care, the community-fostered model is likely more cost-effective compared to traditional models of scent detection dog preparation. Not only are there economic benefits to the organization in 
using this approach, such a model strategically distributes resources and makes the most of professional trainers' time and expertise. By having fundamental manners/obedience training introduced by the puppy's foster family, professional trainers can focus on training specialized skills, such as odor detection, which may require expert knowledge and/or equipment.

Once again, although the relative success rate of dogs from a community-fostered model is unknown, it may be the case that overall wastage (time, resources, and failure for dogs to succeed) in the program is reduced. Compared to a more traditional model, unsuccessful detection dogs in the program may more easily be adopted due to their previous experience living in a home and fundamental manners/obedience training. The external socialization practices and intensive regimented training may not only increase the time available for specialized training, ultimately resulting in more successful dogs, but also reduce the wastage of time and financial resources invested into unsuccessful dogs.

\section{Shelter-Based Models}

The last nonconventional approach we discuss as a part of this commentary is what we term the shelterbased model. This model of preparing and deploying scent detection dogs is one in which organizations select and train dogs sourced from animal shelters or rescue organizations. Typically, organizations evaluate potential dogs based on key characteristics/traits suggested to be important for scent detection work, such as "energetic" and "excessive play drive" (Conservation Canines, n.d.; for a review of identifying suitable detection dogs, see Jamieson, Baxter, \& Murray, 2017). Most often, candidate dogs are adopted by the organization and are housed and trained at a designated training facility, in which experts or trainers handle deployment. Generally, this approach is integrated within nonprofit organizations that may, or may not, be affiliated with a parent organization such as a university or research institution. Approaches classified under this shelterbased model operate in a variety of ways. Some operate in a consulting capacity to aid project development, which may ultimately extend to include dog-handler deployment. Others incorporate individual dog and/or handler leases, where a trained dog or handler is hired individually instead of as a dog-handler team. Organizations that have implemented these shelter-based approaches include, but are not limited to, Working
Dogs for Conservation, Conservation Canines, and PACKLEADER.

\section{Benefits to the Dogs and Organizations}

Various benefits exist in adopting the shelter-based approach, particularly to the dogs selected. The shelterbased model frequently emphasizes a "second chance to high-drive shelter dogs" (Working Dogs for Conservation, 2015), as they may be "difficult to maintain as family pets" (Conservation Canines, n.d.). It is often stressed that the dogs sourced for such programs may "have been euthanized if we had not put them to work" (Working Dogs for Conservation, 2015). Therefore, one benefit to this nonconventional approach is that a dog, potentially not suitable for an average home, may be able to thrive and satisfy their energetic needs within this organizational structure.

Many potential costs are associated with other models of scent detection preparation and deployment, such as buying, breeding, and raising dogs. By identifying key characteristics that make for "good detection dogs" (Conservation Canines, n.d.; Working Dogs for Conservation, 2015), and sourcing ideal candidates from shelters and rescues, organizations can minimize their associated expenses. Breeding programs can be costly to implement and maintain, especially considering that these costs extend from the acquisition of the dog through development, and necessary staff are required to raise and socialize puppies. By acquiring already reared candidates from shelters or rescues, some of these associated costs may be reduced, and relevant breeding and puppy-raising programs offset as shelter adoption fees may be lower in cost than fees for sourcing, purchasing, and/or breeding for a specific candidate. Furthermore, organizations sourcing dogs from a shelter or rescue have a pool of dogs of varying ages and development. An organization wishing to train a dog that has high persistence and interest in toys may be able to choose a dog that already demonstrates these traits rather than attempting to use predictive assessments with a younger puppy.

Although the relative success rate of dogs from a shelter-based model is unknown, organizations implementing this approach are likely more cost-effective compared to traditional models of scent detection dog preparation. The reduced costs associated with preparing and kenneling, particularly during the early years of a dog's life, may offset the costs of housing and caring for dogs during training and after successful completion 
of the training program. Ultimately, both dogs and organizations may benefit from the implementation of this model, as behaviors that may be undesirable for a pet or companion may be exceptionally desirable for a successful detection dog.

\section{Conclusions}

The target article by Troisi et al. (2019) provides a foundational summary of relevant research for scentdetection preparation and deployment procedures. However, the focus lends itself to a more traditional model in which dogs are bred and/or selectively purchased and are reared, trained, and housed at their respective organizations for preparation and deployment. In an effort to "encourage the co-operation and synergy between academics interested in the purer aspects of animal cognition and professionals interested in either preparation and deployment of dogs for scent detection tasks" (Troisi et al., 2019, p. 52), we add to the target article by highlighting nonconventional approaches of scent detection dog preparation and deployment that may have various benefits to both the dog and the organization. Although specific benefits relating to the dogs and organizations have been discussed earlier in this commentary, we conclude by emphasizing that this variation may support greater variability of handler skills and dog temperaments, which in turn can provide additional benefits to the scent detection community. We stress that by evaluating nonconventional models, researchers may better understand the ideal qualities of a detection dog-handler team. Cooperation between academics and industry professionals may ultimately result in benefits for all stakeholders involved.

We acknowledge that there may be limitations to the preparation and deployment of nonconventional approaches, yet we insist that there are benefits to the dog, the organization, and researchers interested in evaluating various effects underlying the success of scent detection dogs. It is important to note, however, that given variation in the use and implementation of preparation and deployment procedures across both biological and nonbiological scent detection training that nonconventional approaches may not be suited for certain types of detection training. For example, it is possible that some nonconventional approaches to preparation and deployment procedures may not be suited for high-risk detection work (e.g., explosives detection). It may be the case that under certain circumstances, specific, regimented training structures are necessary and, in fact, deliver the highest benefit to both the dogs and the organizations. Perhaps the nonconventional models just discussed are best suited to be implemented complementary to existing traditional models, expanding opportunities beyond those currently filled by organizations using traditional training and deployment practices. Questions like this remain unanswered and available for future exploration.

\section{References}

Beebe, S. C., Howell, T. J., \& Bennett, P. C. (2016). Using scent detection dogs in conservation settings: A review of scientific literature regarding their selection. Frontiers in Veterinary Science, 3, 96. doi:10.3389/fvets.2016.00096

Browne, C., Stafford, K., \& Fordham, R. (2006). The use of scent-detection dogs. Irish Veterinary Journal, $59,97-104$.

Cobb, M., Branson, N., McGreevy, P., Lill, A., $\&$ Bennett, P. (2015). The advent of canine performance science: Offering a sustainable future for working dogs. Behavioural Processes, 110, 96-104. doi:10.1016/j.beproc.2014.10.012

Conservation Canines. (n.d.). Center for Conservation Biology. Retrieved from https://conservationbiology.uw.edu /conservation-canines/

Evans, R. I., Herbold, J. R., Bradshaw, B. S., \& Moore, G. E. (2007). Causes for discharge of military working dogs from service: 268 cases (2000-2004). Journal of the American Veterinary Medical Association, 231, 1215-1220.

Jamieson, L. T. J., Baxter, G. S., \& Murray, P. J. (2017). Identifying suitable detection dogs. Applied Animal Behaviour Science, 195, 1-7. doi:10.1016/j.applanim.2017.06.010

Jamieson, L., Baxter, G., \& Murray, P. (2018). You are not my handler! Impact of changing handlers on dogs' behaviours and detection performance. Animals, 8, E176. 
Maejima, M., Inoue-Murayama, M., Tonosaki, K., Matsuura, N., Kato, S., Saito, Y., . . . Ito, S. (2007). Traits and genotypes may predict the successful training of drug detection dogs. Applied Animal Behaviour Science, 107, 287-298. doi:10.1016/j.applanim.2006.10.005

New Zealand Department of Conservation. (n.d.). Conservation Dogs Programme. Retrieved from https://www.doc.govt.nz/our-work/conservation -dog-programme/

Penn Vet. (n.d.). Penn Vet Working Dog Center. Retrieved from http://www.vet.upenn.edu/research /centers-initiatives/penn-vet-working-dog-center

Rooney, N. J., Clark, C. C. A., \& Casey, R. A. (2016). Minimizing fear and anxiety in working dogs: A review. Journal of Veterinary Behavior, 16, 53-64. doi:10.1016/j.jveb.2016.11.001
Troisi, C. A., Mills, D. S., Wilkinson, A., \& Zulch, H. E. (2018). Behavioral and cognitive factors that affect the success of scent detection dogs. Comparative Cognition \& Behavior Reviews, 14, 51-76. doi:10.3819/CCBR.2019.140007

Wilsson, E., \& Sundgren, P.-E. (1997). The use of a behaviour test for selection of dogs for service and breeding. II. Heritability for tested parameters and effect of selection based on service dog characteristics. Applied Animal Behaviour Science, 54, 235-241. doi:10.1016/S0168-1591(96)01175-6

Working Dogs for Conservation. (2015). Our work. Retrieved from https://wd4c.org/ourwork.html 